Centre for Evidence Based Medicine, Nuffield Department of Primary Care Health Sciences, University of Oxford, Oxford

jeffrey.aronson@phc.ox.ac.uk Cite this as: BMJ 2021;375:n2934 http://dx.doi.org/10.1136/bmj.n2934 Published: 26 November 2021

\section{When I use a word .... No jab, no job? A benefit:harm balance analysis}

The current debate about whether individuals should be entitled to work in the healthcare sector if they decline to be vaccinated against SARS-CoV2 has been largely informed by personal opinions and argument by analogy. A benefit:harm balance analysis suggests that while vaccination has a highly favourable benefit:harm balance, the balance in instituting a "no jab, no job" policy is highly uncertain and may be unfavourable. Furthermore, there are practical difficulties and legal uncertainties. The much misunderstood precautionary principle dictates that if the benefit:harm balance of an intervention is unclear and may be unfavourable, the intervention should not be undertaken. Furthermore, the onus is on those who believe that the benefit:harm balance will be favourable to prove that it is so; it is not for the sceptics to prove that it isn't. In the absence of good evidence in favour, this is an intervention that would be best avoided.

Jeffrey K Aronson,

Since the SARS-CoV2 vaccines were developed, the question of whether a policy of encouraging healthcare workers to undergo vaccination, in the absence of medical contraindications, by otherwise declining to employ them in frontline healthcare, has been the subject of a great deal of discussion, including personal opinion, sometimes tinged with emotion, and argument by analogy. Many countries have now made this mandatory for healthcare staff, including NHS staff in England, who will have to be vaccinated by 1 April 2022. ${ }^{1}$ Such discussions are by no means new, and healthcare workers are already required or encouraged to undergo some forms of vaccination against diseases such as hepatitis B as part of their employment, mandatory in some countries and recommended in others, ${ }^{23}$ including the UK. 45

Every intervention we undertake in healthcare is undertaken in the expectation that it will bring benefit. Furthermore, the chance of benefit from the intervention should outweigh the possibility of harm, which can never be ruled out. ${ }^{6}$ However, failing to introduce an intervention can also lead to harm.

In order to assess whether to introduce an intervention, an analysis of the benefit to harm balance is necessary. Three questions inform the assessment:

1. What is the chance of benefit from the intervention?

2. What is the chance of harm from the intervention?

3. What is the chance of harm from not intervening?

When assessing these chances, the nature of the benefits and harms should be taken into account as well as the raw probabilities. So too should the availability of other interventions, which might be equally or more beneficial and less harmful. When considering any intervention in clinical practice in general, the answers to these questions should ideally come from large randomised population studies.

Assessing the benefit:harm balance in an individual is more difficult because one rarely knows in advance what the likely individual response will be. Each occasion of intervention is, in effect, an experiment.
That is why it is generally a good pharmacological principle, when embarking on a course of treatment, to start with a low dose of a medicine, carefully increasing the dose while monitoring for beneficial and adverse effects. But for one-off treatments, like vaccination, one has to rely on expectations from population studies.

What do we find when we subject the intervention of vaccination against SARS-CoV2 and the policy of "no jab, no job” to benefit:harm balance analyses?

\section{Intervention A. SARS-CoV2 vaccination}

1: Chance of benefit-very high, since there is a high rate of protection from infection, especially severe infection. ${ }^{7}$

2. Chance of harm-very low: mild adverse effects of vaccination are common but transient and sometimes associated with a nocebo effect ${ }^{8}$; serious adverse effects are rare. ${ }^{9}$

3. Chance of harm from not intervening-quite high, since the adverse effects of the infection are more common and more severe than the adverse effects of vaccination.

Interpretation: a highly favourable benefit:harm balance, supporting vaccination.

\section{Intervention B. The policy of "no jab, no job"}

1: Chance of benefit-high, if it encourages staff to be vaccinated.

2. Chance of harm-unknown; there are two possible outcomes, discussed below.

3. Chance of harm from not intervening-quite high, since staff will be at risk of infection if the policy is not implemented, leading to absenteeism and possible loss of staff through severe infection; unvaccinated staff may also increase the risk of infections among coworkers and patients. ${ }^{10}$

The uncertainty associated with the question of whether implementation of the policy will be harmful has two main aspects. First, staff may leave because they value their right not to be vaccinated more than they value their jobs; such individuals may already 
be considering leaving, because of increased pressure at work. Secondly, the policy may encourage some who were wavering over vaccination to be vaccinated. In either case the proportion of vaccinated staff will increase, which might be regarded as a benefit. But, even so, if the number of staff is reduced as a result, there will be even more pressure on the service to deliver healthcare. In addition to this, some of those who decide to be vaccinated will suffer adverse effects of the vaccine, although few if any will suffer severe reactions. And some may be aggrieved that they felt constrained to be vaccinated.

Some of these uncertainties could be resolved by a cluster-randomised trial, in which the policy would be introduced in some areas and not others. However, it is unlikely that such a study will be carried out, there being at least two major problems. First, randomised trials of social policies are not in the repertoire of government. Others sometimes carry out trials of therapeutics policies, but it is hard to find good examples in UK practice, and there is some evidence that not all GPs are in favour of such trials. ${ }^{11}$ Secondly, it is likely that those who find themselves in an area in which the policy has been instituted would object that they have been unfairly picked upon, causing resentment, even though the choice was randomised.

There are other practical difficulties. The legality of dismissing someone from their post because of a refusal to accept vaccination, because, for example, of religious scruples or severe anxiety about the possibility of adverse effects, is dubious and could well be challenged in an unfair dismissal tribunal, with uncertain outcomes. Laying them off would be expensive, and redeployment to non-frontline posts might not be possible. Equally, the enforceability of any contract signed by incoming staff, agreeing to undertake vaccination but followed by a change of heart, is hard to judge.

Attractive though it may appear, this is not a policy that seems likely to be justifiable in the NHS. The benefit:harm balance is too uncertain and there are major impracticalities. We can wait to see what happens in countries in which a similar policy has been introduced, but there will always be doubts about the transferability of such outcomes to the UK. Evidence may also come from the care home sector, in which the policy was introduced on 11 November. ${ }^{12}$

If the policy is introduced without a randomised trial, whatever the outcome, some will claim that it was beneficial, others that it was harmful, depending on their preconceived prejudices. Some will adduce different forms of evidence and claim that piling weak evidence upon weak evidence upon weak evidence produces strong evidence in one direction or another. We have seen this happen, for example, in relation to the purported efficacy of some pharmacological interventions during the pandemic, with harmful outcomes.

In making this decision the much misunderstood precautionary principle is helpful. It states that if the benefit:harm balance of an intervention is unclear and may be harmful, the intervention should not be undertaken. Furthermore, the onus is on those who believe that the benefit:harm balance will be favourable to prove that it is so, not for the sceptics to prove that it isn't. In the absence of good evidence in favour, this is an intervention that is best avoided.
3 De Schryver A, Lambaerts T, Lammertyn N, François G, Bulterys S, Godderis L. European survey of hepatitis B vaccination policies for healthcare workers: An updated overview. Vaccine 2020;38:2466-72. doi: 10.1016/j.vaccine.2020.02.003 pmid: 32057571

4 Ramsay M (editor). In: Immunisation Against Infectious Diseases. Chapter 12. Immunisation of healthcare and laboratory staff. Department of Health and Social Care, https://assets.publishing.service.gov.uk/government/uploads/system/uploads/attachment_data/file/147882/GreenBook-Chapter-12.pdf.

5 Komatsu H, Klenerman P, Thimme R. Discordance of hepatitis B vaccination policies for healthcare workers between the USA, the UK, and Germany. Hepatol Res 2020;50:272-82 doi: 10.1111/hepr.13470 pmid: 31845478

6 Smith R. Think harm always. BMJ 2004; 329: 0-g. https://www.bmj.com/content/329/7456/0.8.

7 Rotshild V, Hirsh-Raccah B, Miskin I, Muszkat M, Matok I. Comparing the clinical efficacy of COVID-19 vaccines: a systematic review and network meta-analysis. Sci Rep 2021;11:22777. doi: 10.1038/s41598-021-02321-z pmid: 34815503

8 Amanzio M, Mitsikostas DD, Giovannelli F, Bartoli M, Cipriani GE, Brown WA. Adverse events of active and placebo groups in SARS-CoV-2 vaccine randomized trials: A systematic review[Online ahead of print.]. Lancet Reg Health Eur 2021;0:100253.

doi: 10.1016/j.lanepe.2021.100253. pmid: 34729549

9 Pormohammad A, Zarei M, Ghorbani S, etal. Efficacy and safety of COVID-19 vaccines: a systematic review and meta-analysis of randomized clinical trials. Vaccines (Basel) 2021;9:467 doi: 10.3390/vaccines9050467 pmid: 34066475

10 Abbas M, Robalo Nunes T, Martischang R, etal. Nosocomial transmission and outbreaks of coronavirus disease 2019: the need to protect both patients and healthcare workers. Antimicrob Resist Infect Control 2021;10:7. doi: 10.1186/s13756-020-00875-7 pmid: 33407833

11 Mackenzie IS, Wei L, Paterson KR, Macdonald TM. Cluster randomized trials of prescription medicines or prescribing policy: public and general practitioner opinions in Scotland. Br J Clin Pharmacol 2012;74:354-61. doi: 10.1111/j.1365-2125.2012.04195.x pmid: 22288609

12 Department of Health and Social Care. Coronavirus (COVID-19) vaccination of people working or deployed in care homes: operational guidance. 19 October 2021. https://www.gov.uk/government/publications/vaccination-of-people-working-or-deployed-in-care-homes-operational-guid ance/coronavirus-covid-19-vaccination-of-people-working-or-deployed-in-care-homes-operationalguidance. 